Muro de la Investigación, 2021(1), enero-junio ISSN: 2523-2886

Doi: https://doi.org/10.17162/rmi.v6i1.1439

\title{
Eficiencia del método de lombrifiltro en la remoción de los contaminantes de las aguas residuales domésticas
}

\section{Efficiency of the earthworm method in the removal of pollutants from domestic wastewater}

\author{
Xiomi Vasni Saboya Rios ${ }^{1 \mathrm{a}}$ \\ Universidad Peruana Unión, Lima-Perú ${ }^{1}$
}

Recibido: 07 de octubre de 2020

Aceptado: 15 de diciembre de 2020

\section{Resumen}

El presente estudio buscó determinar la eficiencia de la lombriz de tierra (Lumbricus terrestris) y la lombriz roja californiana (Eisenia foetida) en el tratamiento de las aguas residuales del Distrito de Chachapoyas (Perú). Para ello, se aplicó la adaptación de las dos especies durante un periodo de siete días, luego se inocularon al sistema del lombrifiltro conformado por dos estructuras de madera con cuatro capas, y finalmente se empleó un tanque de almacenamiento de flujo continuo. A fin de determinar la eficiencia de remoción de las especies, se analizó la cantidad de concentración de los parámetros fisicoquímicos y microbiológicos del agua residual que ingresó al proceso de tratamiento. Obteniendo los resultados que la especie EF redujo en un 92\% la DBO5, 86\% DQO, 78\% NT, $84.4 \%$ turbidez, $93 \%$ pH y $84 \%$ en CT a diferencia de la especie LT que obtuvo una reducción del $91 \%$ de DBO5, $84 \%$ DQO, $77 \%$ NT, $83 \%$ turbidez, $93 \%$ pH y $80 \%$ en CT. Se concluyó que la especie EF es más eficiente en la remoción de los contaminantes del agua residual doméstica con un promedio de $87 \%$ en comparación con la especie LT que logró un $85 \%$ siendo aceptables por el DS N 004-2017-MINAM.

Palabras claves: Lombrifiltros, Eisenia foetida, Lumbricus terrestris, microbiología, DBO, DQO, Amazonas.

\begin{abstract}
The present study determined the efficiency of the earthworm (Lumbricus terrestris) and the Californian red earthworm (Eisenia foetida) in the treatment of wastewater from the Chachapoyas District. The adaptation of the two species was applied during a period of seven days, then they were inoculated to the worm-filter system made up of two wooden structures with four layers, and finally a continuous flow storage tank. To determine the removal efficiency of the species, the amount of concentration of the physicochemical and microbiological parameters of the wastewater that entered the treatment process was analyzed. Obtaining the results that the EF species reduced BOD5 by $92 \%, 86 \%$ COD, $78 \%$ NT, $84.4 \%$ turbidity, $93 \% \mathrm{pH}$ and $84 \%$ in CT, unlike the LT species, which obtained a $91 \%$ reduction in BOD5. , 84\% COD, $77 \%$ NT, $83 \%$ turbidity, 93\% pH and $80 \%$ in CT. Concluding that the EF species is more efficient in the removal of pollutants from domestic wastewater with an average of $87 \%$ compared to the LT species that achieved $85 \%$ being acceptable by DS $\mathrm{N}^{\circ} 004-2017-$ MINAM.
\end{abstract}

${ }^{\mathrm{a}}$ Correspondencia al autor:

E-mail: xiomisaboya@upeu.edu.pe 
Keywords: Earthworm, Eisenia foetida, Lumbricus terrestris, DBO, DQO

\section{Introducción}

Actualmente, las causas que originan el limitado de TAR se derivan por la falta de educación ambiental; este término se conceptualiza como conductas humanas que deben estar encaminadas vía el desarrollo sostenible, sin comprometer las necesidades de las generaciones futura (Ministerio del Ambiente [MINAM], 2012), en consecuencia, el $60 \%$ de la población peruana terminan descargando estas aguas a los suelos, y a los cuerpos de agua (ríos, lagos, mares), entre otras, (Brack et al., 2008)

Asimismo, las industrias también son responsables de que estas aguas no se traten adecuadamente debido a que sobrepasan las cargas orgánicas aceptables para las plantas de tratamiento de aguas residuales (PTAR); otro tema se relaciona con las empresas prestadoras de servicio (EPS), el 30\% tienen un mantenimiento incorrecto de sus plantas, además de una insuficiente capacidad financiera que no cubren para que todas las aguas residuales sean tratadas; al mismo tiempo se menciona la limitada fiscalización de los vertimientos de las aguas residuales por parte de las autoridades y organismos competentes que trae consigo una inadecuada gestión sobre estos temas (SUNASS, 2014).

A consecuencia de estos actos se producen aguas residuales, lo que trae como efecto el incremento de enfermedades, principalmente gastrointestinales, cutáneas; además de las alteraciones de la capa freática del suelo, eutrofización, aguas subterráneas contaminadas a causa de la infiltraciones de la aguas residuales al suelo, la producción de alimento contaminado a raíz del regadío con aguas no tratadas y la ausencia del preciado recurso para las actividades de subsistencia como la agricultura y ganadería y para el propio ser humano (Lorenzo, et al., 2009).

Dadas las condiciones que anteceden, en la actualidad existen métodos y mecanismos para el tratamiento de aguas residuales, en la cuales se encuentran los sistemas biológicos; estos son métodos que utilizan microorganismos para realizar la degradación biológica de la materia orgánica del agua en ambientes aerobios y anaerobios (Nodal, 2001). Además, existen tipos de sistemas bilógicos como los convencionales y no convencionales; cada uno de estos sistemas poseen métodos, como las lagunas de estabilización, lombrifiltros, filtros percoladores, Biodiscos, lodos activados, lagunas aireadas, entre otros (Pérez, 2010). 
De esta manera, el método del lombrifiltro es considerado un biofiltro cuyos principales degradadores de la materia orgánica son las lombrices, en este biofiltro ingresa toda el agua residual para su posterior tratamiento. Todo el proceso está compuesto por capas o estratos filtrantes de varios materiales, sin embargo, la lombriz y un gran número de microorganismos se encargan de absorber y digerir la materia orgánica dejando el agua sin sus principales contaminantes que son los residuos patógenos presente en el agua residual (Ramón, Leon, \& Castillo, 2015).

En el distrito de Chachapoyas de la Provincia de Chachapoyas, Departamento de Amazonas, las aguas residuales domésticas no son tratadas adecuadamente, en las ultimas supervisiones del Organismo de Evaluación y Fiscalización Ambiental (OEFA, 2016) mediante el informe $\mathrm{N}^{\circ}$ 0459-2016-OEFA-DS-SEP indican que al ser un distrito pequeño no manejan los temas y componentes ambientales apropiadamente, sobre todo en los anexos y centros poblados, debido a que estas no tienen un sistema de alcantarillado, es por esto, que sus aguas residuales domésticas desembocan directamente al río Sonche efluente del río Utcubamba; asimismo se verificó que estas aguas residuales son utilizadas para el riego de vegetales y uso en la ganadería, exponiendo un peligro para la salud de la población y medio ambiente; motivo por el cual se requiere emplear una alternativa de tratamiento de aguas residuales mediante el lombrifiltro, debido a que es un método eficiente, fácil en su manejo y económico, apta para una población que es pequeña y las viviendas están lejanas una de otra y de esa manera se pueda obtener el embellecimiento de las áreas verdes y a la par procuraría mostrar la realidad de fomentar la ecoeficiencia y el desarrollo sostenible de este recurso. Frente a ello este articulo tiene el objetivo de determinar la eficiencia de tratamiento mediante el método del lombrifiltro, en la remoción de los contaminantes de las aguas residuales domésticas del Distrito de Chachapoyas-Amazonas.

\section{Materiales y métodos}

Mediante el siguiente diagrama (Figura 1), se presenta la metodología del proceso de tratamiento de agua residual doméstica y las actividades previas en la investigación: 


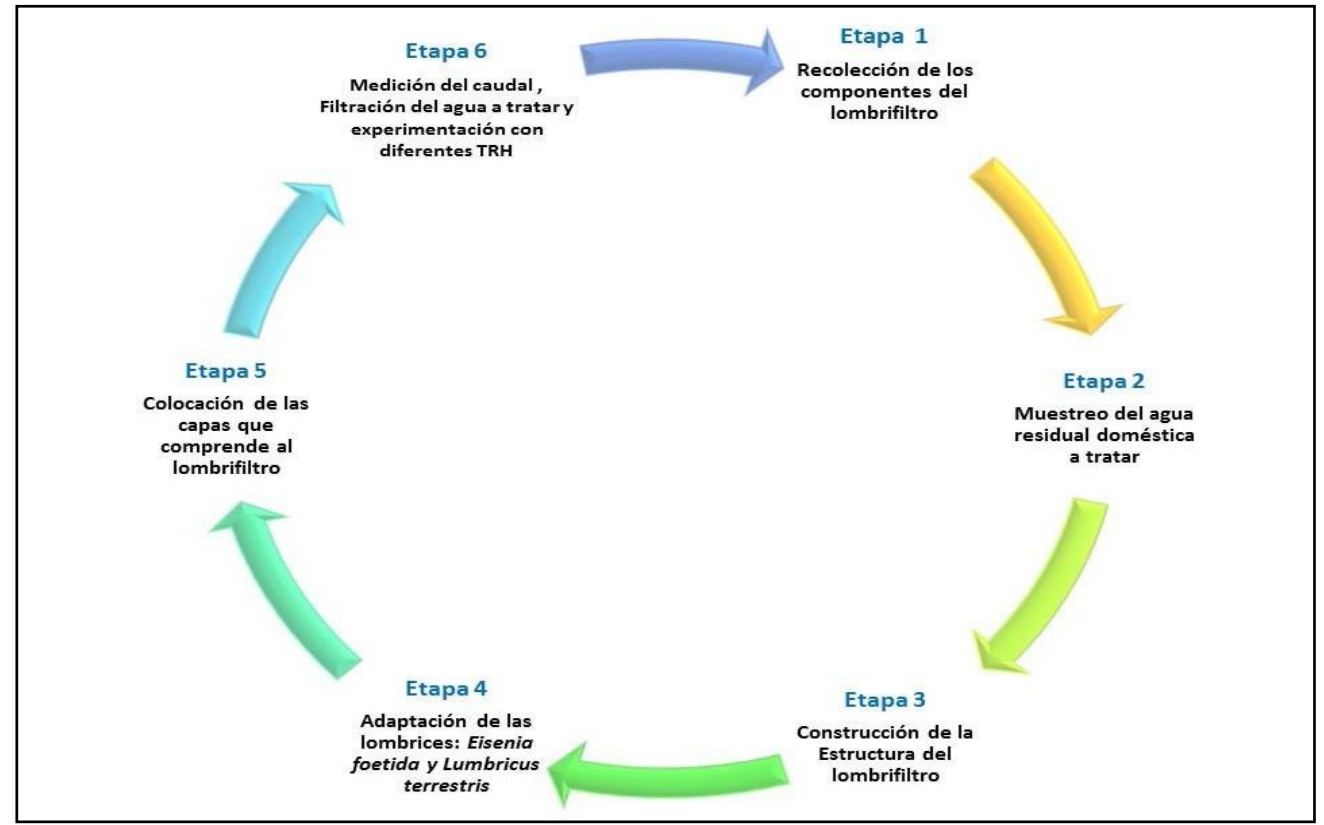

Figura 1. Etapas del proceso de tratamiento de agua residual doméstica, elaboración propia

\section{Recolección de los componentes del Lombrifiltro (Etapa 1)}

\section{Materiales}

Tabla 1

Componentes del Lombrifiltro.

\begin{tabular}{lc}
\hline \multicolumn{1}{c}{ Especie } & Cantidad \\
\hline Eisenia foetida & 1 kilo \\
Lumbricus terrestris & 1 kilo \\
Aserrín & 2 kilos \\
Grava pequeña & 3 kilos \\
Gravas medianas & 3 kilos \\
Piedra de rio enteras & 4 kilos \\
\hline
\end{tabular}

- $\quad$ Rastrillo de jardinería

- $\quad$ Balde

- Bolsa siplot

- $\quad$ Bolsas comunes (negra)

- Guantes 


\section{Método}

Los componentes que se utilizaron, para el lombrifiltro se tomaron de los siguientes lugares según se describe a continuación: Se tomó $1 \mathrm{Kg}$ de Eisenia foetida de las camas de compost que se produce en casa de una agricultora y compostera ubicado en la Av. Grau Cdra. 9 Nº 978 - Chachapoyas. Asimismo, se recogió 1 kg de Lumbricus terrestris, provenientes de un terreno, cerca de un canal de regadío, ubicado en el Jr. Dos de mayo -Chachapoyas.

Para la recolección de ambas especies se realizó una excavación de aproximadamente $30 \mathrm{~cm}$ de profundidad, en una superficie de $1 \mathrm{~m}^{2}$, para luego separarlas de la tierra que se encuentra impregnada en los organismo. Para la obtención del aserrín, se buscó una carpintería ubicado en el Jr. Salamanca $N^{\circ} 458$, además se calculó un aproximado de 2 kilos de aserrín de madera blanca, no es recomendable el uso de aserrín de madera roja por la elevada concentración de lignina, esto porque puede matar a las lombrices. Posteriormente, se pasó a recolectar las piedras de las tres (03) distintas granulometrías con un peso de 3 kilos cada una, excepto la de piedra de rio que se recolecto un total de 4 kilos, de la cantera Jhordan ubicados a la salida de la ciudad de Chachapoyas.

\section{Muestreo del ARD a tratar (Etapa 2)}

\section{Materiales}

- Botella

- Guantes

- Mascarilla

- Balde

- Cooler

- 2 frascos de vidrio claro de $300 \mathrm{ml}$

- 2 frascos de vidrio ámbar de $200 \mathrm{ml}$

- Rotulador o marcador

- Bata

\section{Método}

El (OEFA, 2016), en su Informe de Supervisión del año 2016 menciona que la ciudad de Chachapoyas descarga sus aguas residuales domésticas en los siguientes puntos (Tabla 2): 
Tabla 2

Coordenadas de ubicación de los puntos de descarga de las aguas residuales de la ciudad de Chachapoyas

\begin{tabular}{lccc}
\hline \multirow{2}{*}{ Lugar } & \multicolumn{2}{c}{ Coordenadas } & \multirow{2}{*}{ Zona } \\
\cline { 2 - 3 } & Este & Norte & \\
\hline $\begin{array}{l}\text { Quebrada de Santa Lucia } \\
\text { (descarga al rio Sonche) }\end{array}$ & 183636 & 9311084 & $18 \mathrm{M}$ \\
$\begin{array}{l}\text { Sector Santo Domingo- } \\
\begin{array}{l}\text { El Molino (descarga al } \\
\text { rio Utcubamba) }\end{array}\end{array}$ & 179871 & 9308947 & $18 \mathrm{M}$ \\
\hline
\end{tabular}

Fuente: (OEFA, 2016)

Para esta investigación, se recolectó el agua residual doméstica, de la Quebrada de Santa Lucia, seguidamente se determinó el horario de muestreo, para ello se tomaron muestras en las siguientes horas: 7:00, 12:00 y 17:30 horas; ya que según (Madueño \& Sandoval, 2009), se debe elegir la hora donde existe un mayor valor de DQO, en este caso se evaluó solo vía visual, con el color de agua y carga orgánica que se podía ver en el momento y se eligió el horario de 17:30, esto debido a que es la hora en que toda la población regresan casa, y el uso del agua es mayor, además que las ganaderías y mataderos se hacen presentes en el momento.

\section{Muestreo del ARD para el análisis de los parámetros}

Este proceso se realizó en un tiempo de 6 días, tomando 3 litros diarios, recolectando una muestra total de $18 \mathrm{Lt}$ tal como se muestra en la siguiente tabla 3 :

Tabla 3

Recolección del agua residual domestica

\begin{tabular}{cccc}
\hline $\begin{array}{c}\mathbf{N}^{\circ} \text { Muestras } \\
\text { diarias }\end{array}$ & $\begin{array}{c}\text { Volumen } \\
\text { recolectado } \\
\text { (litros) }\end{array}$ & $\begin{array}{c}\mathbf{N}^{\circ} \text { de días de } \\
\text { muestreo }\end{array}$ & $\begin{array}{c}\text { Volumen total } \\
\text { recolectado } \\
\text { (litros) }\end{array}$ \\
\hline 1 & 3 & 6 & 18 \\
\hline
\end{tabular}

Finalmente se recolectó $30 \mathrm{Lt}$ donde $18 \mathrm{Lt}$ fueron utilizados para el tratamiento, 5 Lt para los análisis respectivos y 7 Lt para el proceso de adaptación del material biológico (Eisenia foetida y Lumbricus terrestris). Para la medición de los parámetros del agua residual doméstica, tanto para el afluente y efluente, se realizó la respectiva desinfección de los recipientes, frascos estériles y su correspondiente homogenización y preservación, para luego ser direccionados al Laboratorio certificado NKAP SAC, para la evaluación 
de los parámetros de $\mathrm{pH}$, coliformes termotolerantes, DBO, DQO, turbidez y nitrógeno total. En cuanto al muestreo:

a) Para la Demanda Química de Oxigeno (DBO) y Demanda Biológica de Oxígeno (DBO), se tomó un frasco de vidrio claro de $300 \mathrm{~mL}$ y se llenó de agua residual hasta el límite para luego agregarle el preservante (H2SO4), y posteriormente fue guardado en el Cooler para enviarlo al laboratorio NKAP.

b) Para los parámetros microbiológicos (Coliformes termotolerantes) la muestra fue tomada en un frasco de vidrio claro de $300 \mathrm{~mL}$, dejando un espacio de $1 / 4$, no se llenó por completo la botella.

c) Para el caso de nitrógeno se utilizaron frascos de vidrio ámbar de $200 \mathrm{~mL}$, se añadió el preservante (H2SO4).

d) Por último, para los análisis físicos (turbidez, $\mathrm{pH}$ ) se utilizó un frasco de vidrio ámbar de $200 \mathrm{~mL}$.

\section{Construcción de la estructura del Lombrifiltro (Etapa 3)}

\section{Materiales}

- Tanque de 140 Lt de capacidad

- Estructura de madera de cinco compartimientos

- Tuberías $1 / 2$ pulgada

- 3 Llaves de control

- 12 Cintillos
- Codos de PVC de $1 / 2$

- 05 recipientes de plásticos

- Silicona y pegamento para tuberías

- Tuercas y desarmadores

\section{Método}

Para su construcción, manipuleo y acceso a los materiales en la zona se elaboró de la siguiente manera:

- Tanque de almacenamiento: Se compró y adaptó un tanque de plástico de 140 Lt, para almacenar y permitir el ingreso a los sistemas el agua residual doméstica de la ciudad de Chachapoyas.

- Fuente de ingreso: Se construyó con tuvo PVC de 1/2”, una llave de ingreso y 2 llave de control de $1 / 2$ " para los dos sistemas, según medida de la estructura de madera.

- Construcción de las estructuras de madera: Se construyeron 2 estructuras de madera, para el tratamiento de las aguas residuales, de la cuales se realizaron 5 compartimentos con dimensiones de $0.25 \mathrm{~m}$ ancho x $0.45 \mathrm{~m}$ largo x $0.30 \mathrm{~m}$ 
profundidad, cada uno conteniendo un recipiente de plástico de dimensiones $0.25 \mathrm{~m}$ x ancho $0.45 \mathrm{~m}$ largo $\mathrm{x} 0.25 \mathrm{~m}$ profundidad; de las cuales se encuentra en la primera capa el materiales biológicos las especie $E F$ y en la segunda estructura está la especie $L T$ cada uno con aserrín de madera blanca, en la segunda capa se encuentra gravas menudas, en la tercera capa grava chancadas, en la cuarta capa bolones de piedra de rio, estas piedras fueron previamente lavadas y en la última capa se encuentra el recipiente de almacenamiento donde se recoge el agua ya tratada para su posterior control final. Cada compartimento tiene $5 \mathrm{~cm}$ de separación para que exista ventilación entre ellos.

- Asimismo, cada estrato filtrante tiene diferentes dimensiones en la cantidad a incluir en cada capa, las mismas que se propusieron tomando como referencia el estudio de Salazar, 2005 "sugiere que la parte biológica debe contener entre un 60 a 70\%", por lo tanto, se dispuso entre la primera y segunda capa que constituyen el material biológico más elevado será un $60 \%$ Eisenia foetida y las gravas finas o menudas respectivamente, en la tercera capa un $20 \%$ de grava chancada y en la cuarta capa un $20 \%$ de piedras enteras de rio (Tabla 4 ).

Tabla 4

Dimensiones de cada capa de la estructura

\begin{tabular}{ccc}
\hline Descripción & Altura (cm) & Altura (m) \\
\hline Eisenia foetida + aserrín & 26 & 0.26 \\
Lumbricus terrestris+ aserrín & 13 & 0.13 \\
Grava fina & 13 & 0.13 \\
Grava chancada & 13 & 0.13 \\
Piedras de rio &
\end{tabular}

\section{Adaptación de las lombrices: EF y LT (Etapa 4)}

Antes del tratamiento de filtración, se realizó un proceso de adaptación, especialmente de la lombriz, para poder tener un correcto tratamiento del agua residual. Esto debido a que es un ser vivo y necesita la adecuación al proceso que se va a llevar a cabo, y sobre todo porque el agua tiene contaminantes y las lombrices van estar expuestas a estos.

\section{Materiales}

- Guantes

- Malla tipo raschell
- Regadera

- Balanza 
- 2 cajas de madera

\section{Método}

El procedimiento que se realizó para la adaptación de la Eisenia foetida y Lumbricus terrestris, se llevó a cabo en dos ensayos etiquetados cada uno respectivamente como T1 y $\mathrm{T} 2$. En cada recipiente respectivamente se realizaron agujeros y se colocó una malla tipo Raschell en el fondo de los recipientes. Luego se preparó el sustrato dentro de cada recipiente, para ello se aspergeó $1000 \mathrm{ml}$ de agua residual para la tierra y aserrín, se colocó una capa de sustrato preparado y, se realizó la prueba de puño para verificar el porcentaje de humedad; y se dio la inoculación de las lombrices en cada recipiente; este proceso de adaptación duró 7 días (tabla 5 y 6).

Tabla 5

Adaptación de Eisenia foetida

\begin{tabular}{cccccc}
\hline \# De Dias & Fecha & Hora & Temperatura $^{\circ} \mathbf{C}$ & Humedad \% & Peso \\
\hline 2 & $02 / 10 / 2017$ & $9: 00$ & 19.8 & 80 & $511 \mathrm{~g}$ \\
2 & $02 / 10 / 2017$ & $18: 00$ & 9.4 & 80 & $515 \mathrm{~g}$ \\
7 & $07 / 10 / / 2017$ & $9: 00$ & 18.8 & 80 & $586 \mathrm{~g}$ \\
7 & $07 / 10 / 2017$ & $18: 00$ & 9.8 & 80 & $594 \mathrm{~g}$ \\
\hline
\end{tabular}

Tabla 6

Adaptación de Lumbricus terrestris

\begin{tabular}{cccccc}
\hline \# De Días & Fecha & Hora & Temperatura $^{\circ} \mathbf{C}$ & Humedad \% & Peso \\
\hline 2 & $02 / 10 / 2017$ & $9: 00$ & 19.8 & 80 & $508 \mathrm{~g}$ \\
2 & $02 / 10 / 2017$ & $18: 00$ & 9.4 & 80 & $511 \mathrm{~g}$ \\
7 & $07 / 10 / \mid 2017$ & $9: 00$ & 18.8 & 80 & $593 \mathrm{~g}$ \\
7 & $07 / 10 / 2017$ & $18: 00$ & 9.8 & 80 & $614 \mathrm{~g}$ \\
\hline
\end{tabular}

\section{Colocación de las capas del lombrifiltro (Etapa 5)}

\section{Materiales}

- Eisenia foetida

- Lumbricus terrestris

- Aserrín
- Gravas menudas

- Gravas chancadas

- Piedras enteras de rio 


\section{Método}

La instalación de las capas filtrantes se realizó de forma manual para las dos (02) estructuras de madera. Primero se realizaron 36 agujeros en cada uno de los recipientes plantillas y se colocó una malla plástica. Luego de esto se colocaron en cada recipiente diferentes capas como se presenta en la siguiente tabla 7:

Tabla 7

Capas del lombrifiltro

\begin{tabular}{|c|c|c|}
\hline \multicolumn{3}{|c|}{ Capas del lombrifiltro } \\
\hline \multirow{3}{*}{ Capa 1} & Especie & ombriz \\
\hline & T1 -Eisenia foetida & $\begin{array}{c}\text { T2-Lumbricus } \\
\text { terrestris }\end{array}$ \\
\hline & \multicolumn{2}{|c|}{ Filtro biológico } \\
\hline Capa 2 & \multicolumn{2}{|c|}{ Grava de $1 \mathrm{~cm}$ de grosor o menuda } \\
\hline Capa 3 & \multicolumn{2}{|c|}{ Grava de $3 \mathrm{~cm}$ de grosor o chancada } \\
\hline Capa 4 & \multicolumn{2}{|l|}{ Piedras enteras de rio } \\
\hline
\end{tabular}

Medición del caudal, TRH, (Etapa 6)

Materiales

- Cronometro

- Recipiente de $1 \mathrm{~L}$

\section{Método}

Para la medición del caudal se abrió la llave de control de entrada del tanque alimentador y se colocó un recipiente de un (01) litro debajo del aspersor, se recolectó el agua residual doméstica durante un minuto y se retiró el recipiente con el agua recolectada. Posteriormente se midió la cantidad de agua residual recolectada, este proceso se repitió tres veces. Cada tiempo de retención hidráulica (TRH), se midió según el tiempo que tarda en pasar el agua por cada estrato, e inicia su aparición en la base de la última capa, según cada caudal aplicado.

\section{Variables de Estudio}

\section{Variable independiente}

En la investigación las especies utilizadas fueron (Eisenia foetida y Lumbricus terrestris), para el método del lombrifiltro este actuará como variables independientes. 


\section{Variables dependientes}

Las variables dependientes a evaluar son los parámetros a ser medidos para la verificación de la calidad del agua residual, estos son los siguientes:

- DBO5

- DQO

- Nitrógeno total
- Coliformes termotolerantes

- Turbidez

- $\mathrm{pH}$

\section{Formulación de hipótesis}

- H1: El método del lombrifiltro con la especie Eisenia foetida es eficiente en la remoción de contaminantes fisicoquímicos y microbiológicos del agua residual doméstica.

- H2: El método del lombrifiltro con la especie Lumbricus terrestris es eficiente en la remoción de contaminantes fisicoquímicos y microbiológicos del agua residual doméstica

- H3: El método del lombrifiltro con la especie Eisenia foetida difiere de la especie Lumbricus terrestris en la remoción de contaminantes fisicoquímicos y microbiológicos del agua residual doméstica.

\section{Estudio y diseño de la investigación}

\section{Tipo y diseño de la investigación}

El presente proyecto está encaminado en una investigación cuantitativa, de diseño pre-experimental, esto, debido a que se van a manipular las variables de estudio (Sampieri, Fernández, y Baptista, 2010), en este caso los parámetros del agua residual; asimismo este tipo de investigación es secuencial la cual sigue una serie de procesos para llegar a la finalidad, y probatorio porque necesita de análisis para poder comprobar que los resultados son ciertos. Además, de la recolección de los datos se fundamenta en la medición de los parámetros fisicoquímicos y microbiológicos, que se lleva a cabo por procedimientos estandarizados.

\section{Análisis estadístico}

Los datos recolectados fueron introducidos y analizados mediante el Software estadístico SPSS 24.0; posteriormente se realizaron análisis descriptivos comparativos para visualizar la diferencia en los resultados de los contaminantes de las AR según las 
especies en estudio. Finalmente el estadístico de contraste de hipótesis experimental utilizado fue el análisis no paramétricos de Wilcoxon para comparar la remoción de los contaminantes fisicoquímicos y microbiológicos de las AR del antes y después de la aplicación del método de lombrifiltro y a su vez la Prueba de U Mann - Whitney para comparar los resultados de la remoción de los contaminantes de la especie Lumbricus terrestris con la Eisenia foetida; estas pruebas se utilizaron por que las muestras analizadas en el estudio fueron pequeñas.

\section{Resultados y discusiones}

\section{Análisis físico-químicos y microbiológicos de los parámetros antes y después del tratamiento}

Los análisis físico-químicos y microbiológicos ( $\mathrm{pH}$, turbidez, DBO5, DQO, nitrógeno total y Coliformes termotolerantes), antes y después del tratamiento mediante el método de lombrifiltro, fueron realizados por el Laboratorio de ensayo Acreditado por el INACAL-DA con registro No LE 026, Cajamarca y Trujillo (ver tabla 8, 9 y 10) asimismo, la comparación de los resultados finales con el D.S Nº04-2017-MINAM (ver Tabla 14).

Tabla 8

Parámetros físico-químicos y microbiológicos antes del tratamiento mediante el método del lombrifiltro

\begin{tabular}{lcccccc}
\hline \multicolumn{5}{c}{ Resultados del Agua Residual (Afluente-Pre-Análisis) } \\
\hline \multirow{4}{*}{ Parámetros } & \multicolumn{4}{c}{ Parámetros Físico-químicos } & Parámetro \\
& $\mathrm{pH}$ & Turbidez & DBO5 & DQO & NT & Microbiológico \\
Resultados & 6.2 & $52.6 \mathrm{NTU}$ & $112.3 \mathrm{mg} / \mathrm{L}$ & $251.4 \mathrm{mg} / \mathrm{L}$ & $68.3 \mathrm{mg} / \mathrm{L}$ & $4200 \mathrm{NMP} / 100 \mathrm{~mL}$ \\
\hline
\end{tabular}

Tabla 9

Parámetros físico-químicos y microbiológicos después del tratamiento mediante el método del lombrifiltro con la especie Eisenia foetida

\begin{tabular}{|c|c|c|c|c|c|}
\hline \multicolumn{6}{|c|}{ Resultados Final del Efluente con la Especie Eisenia foetida) } \\
\hline \multirow[t]{2}{*}{ Parámetros } & $\mathbf{P a}$ & netros F & ico-quín & & $\begin{array}{l}\text { Parámetro } \\
\text { Microbiológico }\end{array}$ \\
\hline & $\mathrm{pH}$ Tur & DBO5 & DQO & NT & $\mathrm{CT}$ \\
\hline Resultados & $6.78 .2 \mathrm{NTU}$ & $9.5 \mathrm{mg} / \mathrm{L}$ & $36.3 \mathrm{mg} / \mathrm{L}$ & $15.3 \mathrm{mg} / \mathrm{L}$ & $685 \mathrm{NMP} / 100 \mathrm{ml}$ \\
\hline
\end{tabular}


Tabla 10

Parámetros físico-químicos y microbiológicos después del tratamiento mediante el método del lombrifiltro con la especie Lumbricus terrestres

\begin{tabular}{ccccccc}
\hline \multicolumn{5}{c}{ Resultados Final del Efluente con la especie Lumbricus terrestris } \\
\hline \multirow{5}{*}{ Parámetros } & \multicolumn{5}{c}{ Parámetros Físico-químicos } & Parámetro \\
& $\mathrm{pH}$ & Turbidez & DBO5 & DQO & NT & Microbiológico \\
Resultados & 6.68 & $9.1 \mathrm{NTU}$ & $10.52 \mathrm{mg} / \mathrm{L}$ & $41.0 \mathrm{mg} / \mathrm{L}$ & $15.7 \mathrm{mg} / \mathrm{L}$ & $820 \mathrm{NMP} / 100 \mathrm{ml}$ \\
\hline
\end{tabular}

Tabla 14

Comparación de los resultados con el Decretos Supremos N 004-2017-MINAM

\begin{tabular}{|c|c|c|c|c|c|}
\hline \multirow[b]{2}{*}{ Parámetro } & \multirow[b]{2}{*}{$\begin{array}{l}\text { Especie } \\
\text { de } \\
\text { lombriz }\end{array}$} & \multirow[b]{2}{*}{$\begin{array}{c}\text { Resultados } \\
\text { de } \\
\text { Laboratorio }\end{array}$} & \multicolumn{3}{|c|}{ 004-2017-MINAM } \\
\hline & & & $\begin{array}{l}\text { Agua para } \\
\text { riego no } \\
\text { restringido }\end{array}$ & $\begin{array}{l}\text { Agua para } \\
\quad \text { riego } \\
\text { restringido }\end{array}$ & $\begin{array}{c}\text { Bebidas } \\
\text { de } \\
\text { animales }\end{array}$ \\
\hline \multirow{2}{*}{ DBO5 } & $\mathrm{EF}$ & $9.5 \mathrm{mg} / \mathrm{L}$ & $15 \mathrm{mg} / \mathrm{L}$ & $15 \mathrm{mg} / \mathrm{L}$ & $15 \mathrm{mg} / \mathrm{L}$ \\
\hline & LT & $10.52 \mathrm{mg} / \mathrm{L}$ & $15 \mathrm{mg} / \mathrm{L}$ & $15 \mathrm{mg} / \mathrm{L}$ & $15 \mathrm{mg} / \mathrm{L}$ \\
\hline \multirow{2}{*}{ (DQO) } & $\mathrm{EF}$ & $36.3 \mathrm{mg} / \mathrm{L}$ & $40 \mathrm{mg} / \mathrm{L}$ & $40 \mathrm{mg} / \mathrm{L}$ & $40 \mathrm{mg} / \mathrm{L}$ \\
\hline & $\mathrm{LT}$ & $41 \mathrm{mg} / \mathrm{L}$ & $40 \mathrm{mg} / \mathrm{L}$ & $40 \mathrm{mg} / \mathrm{L}$ & $40 \mathrm{mg} / \mathrm{L}$ \\
\hline \multirow{2}{*}{ Nitrógeno total } & $\mathrm{EF}$ & $15.3 \mathrm{mg} / \mathrm{L}$ & $100 \mathrm{mg} / \mathrm{L}$ & $100 \mathrm{mg} / \mathrm{L}$ & $100 \mathrm{mg} / \mathrm{L}$ \\
\hline & $\mathrm{LT}$ & $15.7 \mathrm{mg} / \mathrm{L}$ & $100 \mathrm{mg} / \mathrm{L}$ & $100 \mathrm{mg} / \mathrm{L}$ & $100 \mathrm{mg} / \mathrm{L}$ \\
\hline \multirow{2}{*}{ Turbidez } & $\mathrm{EF}$ & $8.2 \mathrm{UNT}$ & $20 \mathrm{UNT}$ & $20 \mathrm{UNT}$ & $20 \mathrm{UNT}$ \\
\hline & $\mathrm{LT}$ & $9.1 \mathrm{UNT}$ & 20 UNT & 20 UNT & $20 \mathrm{UNT}$ \\
\hline \multirow{2}{*}{ pH } & $\mathrm{EF}$ & 6.7 & $6,5-8,5$ & $6,5-8,5$ & $6,5-8,4$ \\
\hline & LT & 6.68 & $6,5-8,5$ & $6,5-8,5$ & $6,5-8,4$ \\
\hline \multirow{2}{*}{$\begin{array}{c}\text { Coliformes } \\
\text { termotolerantes } \\
(\mathrm{NMP} / 100 \mathrm{~mL})\end{array}$} & $\mathrm{EF}$ & 685 & 1000 & 2000 & 1000 \\
\hline & LT & 820 & 1000 & 2000 & 1000 \\
\hline
\end{tabular}

Comparación de parámetros antes y después del tratamiento mediante el método de lombrifiltro con la especie Eisenia foetida y

Lumbricus terrestris

\section{Demanda Biológica de Oxígeno (DBO5)}

En la figura 2 se muestra la diferencia de la DBO5 con el agua residual doméstica que ingresó al lombrifiltro, siendo este valor $112.3 \mathrm{mg} / \mathrm{L}$; y en el efluente (salida) del método de tratamiento con la especie Eisenia foetida la DBO5 obtuvo un resultado de 9.5 $\mathrm{mg} / \mathrm{L}$ con una eficiencia de remoción de este parámetro de $92 \%$ y con la especie Lumbricus terrestris un total de $10.52 \mathrm{mg} / \mathrm{L}$ con una eficiencia de $91 \%$, para ambos casos se redujo notablemente. La eficacia se determinó después de 6 días del inicio del tratamiento, en el primer día se observó el agua aun con cierta turbidez elevada por tal 
razón, se evaluó por día vía visual, obteniendo un resultado óptimo en el sexto día. Esta reducción se debe a la actividad simbiótica de las lombrices y microorganismos aerobios que aceleran y mejoran la descomposición de la materia orgánica esto debido a que las lombrices trabajan como catalizadores biológicos resultantes de las reacciones bioquímicas (Martinez, 2012).

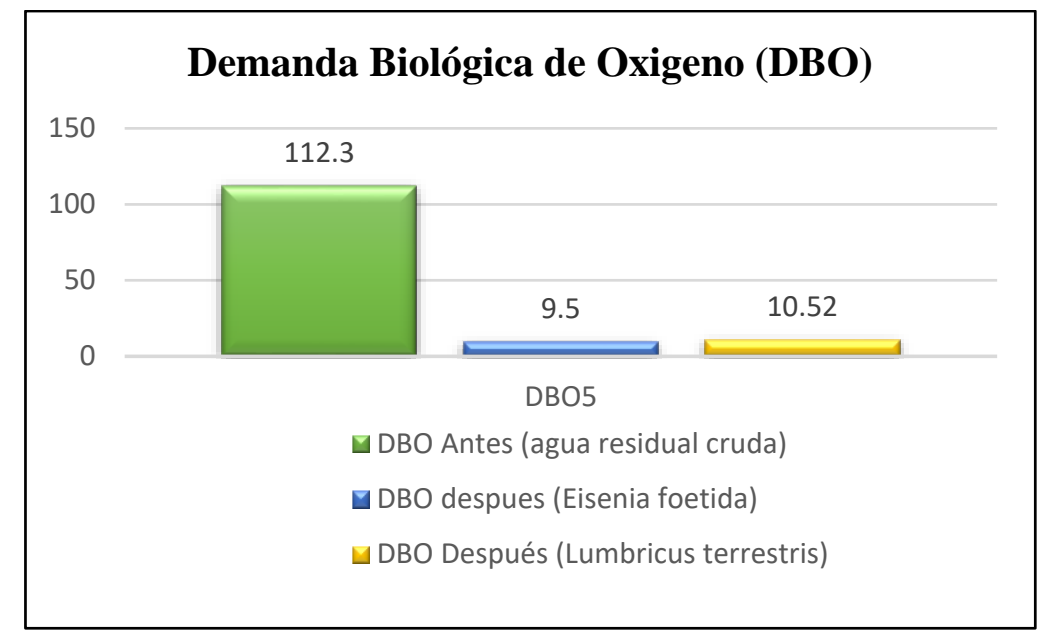

Figura 2. Comparación del parámetro químico DBO5 antes y después del tratamiento mediante el lombrifiltro con las especies Eisenia foetida y Lumbricus terrestris

\section{Demanda Química de Oxígeno (DQO)}

En la figura 3 se muestra la diferencia de la DQO con el agua residual doméstica que ingresó al lombrifiltro, siendo este valor $251.4 \mathrm{mg} / \mathrm{L}$; y en el efluente (salida) del método de tratamiento con la especie Eisenia foetida la DQO obtuvo un resultado de 36.3 $\mathrm{mg} / \mathrm{L}$ con una eficiencia de remoción de este parámetro de $86 \%$ y con la especie Lumbricus terrestris un total de $41 \mathrm{mg} / \mathrm{L}$ con una eficiencia de $84 \%$, para ambos casos hubo una reducción favorable. Esta reducción se puede atribuir a las enzimas en el intestino de las lombrices que contribuyen a la degradación de varios producto químicos, asimismo las lombrices en sus tracto intestinal albergan millones de microbios descomponedores de la materia orgánica e inorgánica, cabe señalar que se observó mayor eliminación en la DBO que en la DQO, esto debido a que las lombrices son principalmente responsables de la biodegradación de los residuos orgánicos en comparación de los residuos inorgánicos.

Por otro lado la reducción del DQO también se debe a la actividad biológica donde ocurren procesos químicos como la oxidación por microorganismos desnitrificadores en condiciones aérobicas, los cuales descomponen todos los compuestos 
orgánicos reduciendo el oxígeno, convirtiéndolo en gas carbónico (CO2) y agua $(\mathrm{H} 2 \mathrm{O})$, donde una parte del $\mathrm{CO} 2$ se pierde en la atmosfera y la otra parte es absorbida por los microorganismos para la síntesis de nuevas células, este proceso depende principalmente de la disponibilidad de oxígeno disuelto, y las lombrices cumplen un papel muy importante en este proceso (Arenas \& Nuncira, 2010)

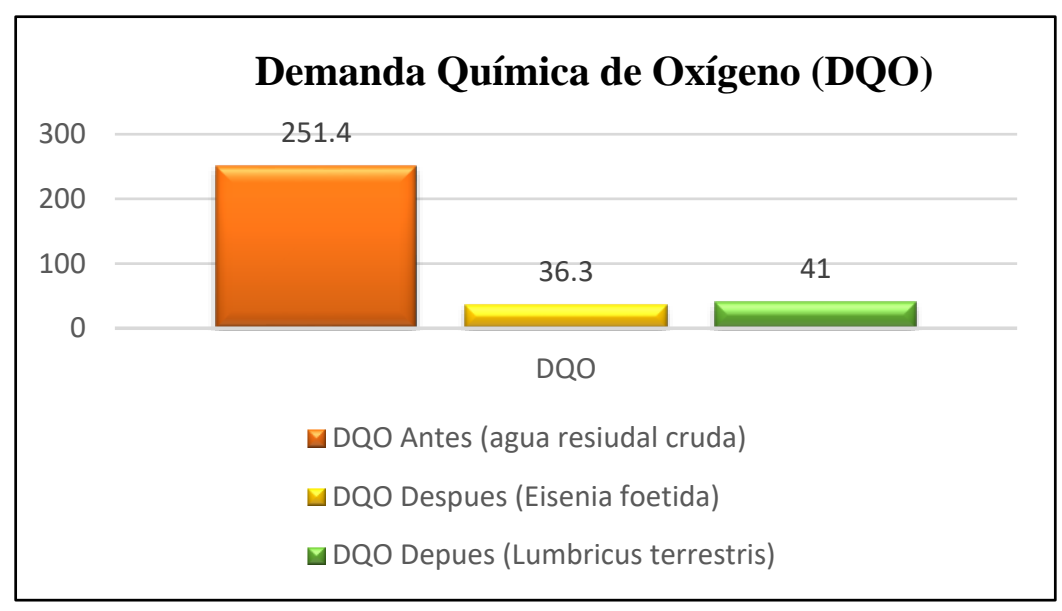

Figura 3. Comparación del parámetro químico DQO antes y después del tratamiento mediante el lombrifiltro con las especies Eisenia foetida y Lumbricus terrestris

\section{Nitrógeno total}

El ingreso del agua residual doméstica con nitrógeno total al lombrifiltro fue de $68.3 \mathrm{mg} / \mathrm{L}$, luego se realizó el análisis respectivo y se obtuvo como resultado con la especie Eisenia foetida un total de $15.3 \mathrm{mg} / \mathrm{L}$ con una eficiencia de $78 \%$; mientras que con la especie Lumbricus terrestris se logró un total de $15.7 \mathrm{mg} / \mathrm{L}$ dando una eficiencia de $77 \%$ (figura 4), notándose una reducción considerable para ambos casos. Esto se atribuye a la mineralización de nitrógeno amoniacal en forma de nitrato debido al impacto directo de las lombrices en las comunidades bacterianas, estas son esencialmente las aeróbicas heterotróficas que son los principales responsables de la mineralización del nitrógeno, asimismo las lombrices secretan polisacáridos, proteínas y otros compuestos nitrogenados, que ayudan en la reducción del nitrógeno en las aguas residuales para que estén en la cantidad disponible para las plantas como nutrientes (Wang et al., 2011).

En comparación, ambas especies son óptimas en la remoción del nitrógeno teniendo una mínima diferencia de $0.4 \mathrm{mg} / \mathrm{L}$, por su naturaleza ambas especies tienen los mismos rasgos al momento de consumir el nitrógeno, sin embargo podemos diferenciar que la especie Eisenia foetida produce más flora microbiana en sus intestinos a diferencia de la Lumbricus terrestris, esto se debe a que la lombriz roja californiana o Eisenia foetida 
suele consumir en cantidad más alimento el mismo o más que su tamaño corporal, además su alimentación se basa en los restos orgánicos (hojas muertas, vegetales en descomposición, heces y animales muertos, etc.) en pocas palabras son de consumir todo tipo de materia orgánica, no obstante la lombriz de tierra común a pesar de tener un tamaño superior a la de la Eisenia foetida solo son de consumir un solo tipo de alimento que consideren propicio para estos.

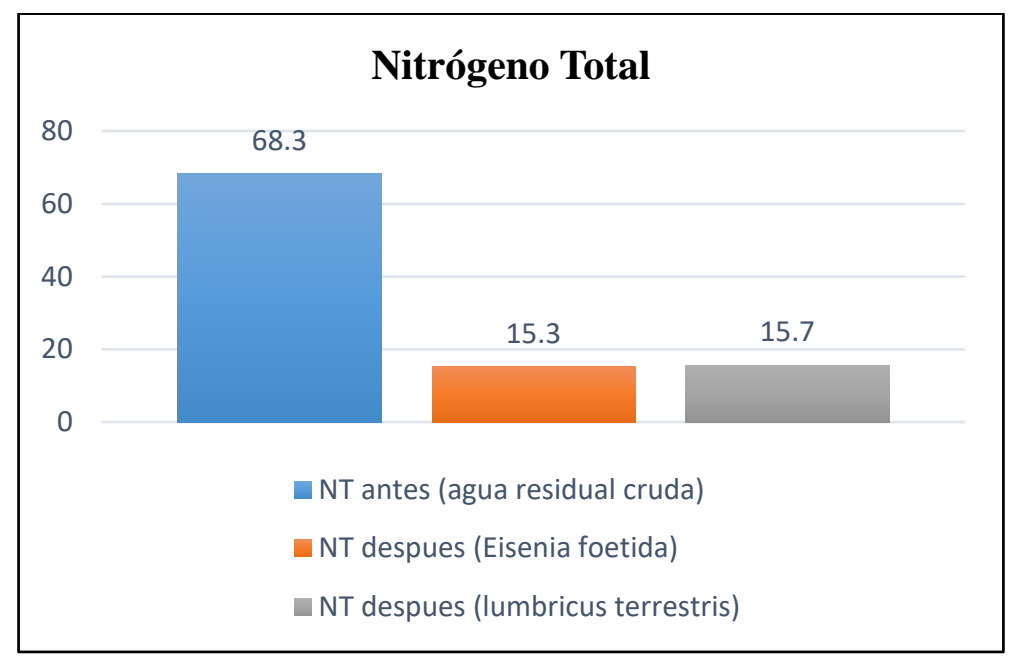

Figura 4. Comparación del parámetro químico Nitrógeno Total antes y después del tratamiento mediante el lombrifiltro con las especies Eisenia foetida y Lumbricus

\section{Turbidez}

El agua residual doméstica ingresó al lombrifiltro con una turbidez de 52.6 unidades nefelométricas de turbidez (UNT), y a la salida del lombrifiltro se obtuvo un total con la especie Eisenia foetida de 8.2 UNT con una eficiencia de $84.4 \%$; de la misma manera con la especie Lumbricus terrestris se obtuvo un total de 9.1 UNT dando una eficiencia de $83 \%$ (figura 5); indicando que el agua residual se encuentra en buen estado, esta variación de turbidez se notó claramente por la transparencia del afluente en comparación con el efluente pasado los 6 días del tratamiento, esto se dio principalmente por las propiedades de las lombrices al convertir los sólidos en su alimento principal y por el medio poroso del filtro.

Es importante recalcar, que ambas especies mostraron resultados aceptables y óptimos en la remoción de la turbidez del agua residual, revelando solo una diferencia mínima de 0.9 UNT. Esta diferencia mayormente está ligada a la temperatura ambiente, 
como se mencionó anteriormente, la especie Lumbricus terrestris es el tipo de lombriz que está acostumbrada vivir en un clima cálido y en suelo profundos por ende son bastante delicados en los cambios bruscos de clima, afectando directamente en su alimentación y reproducción, razón por la cual la especie Eisenia foetida tuvo la ligera ventaja debido a que están acostumbradas a cambios continuos porque al ser de tipo epigeas están constantemente en la superficie de la tierra, no son excavadoras como las Lumbricus terrestris, pero son más adaptables y reproductivas en cantidad.

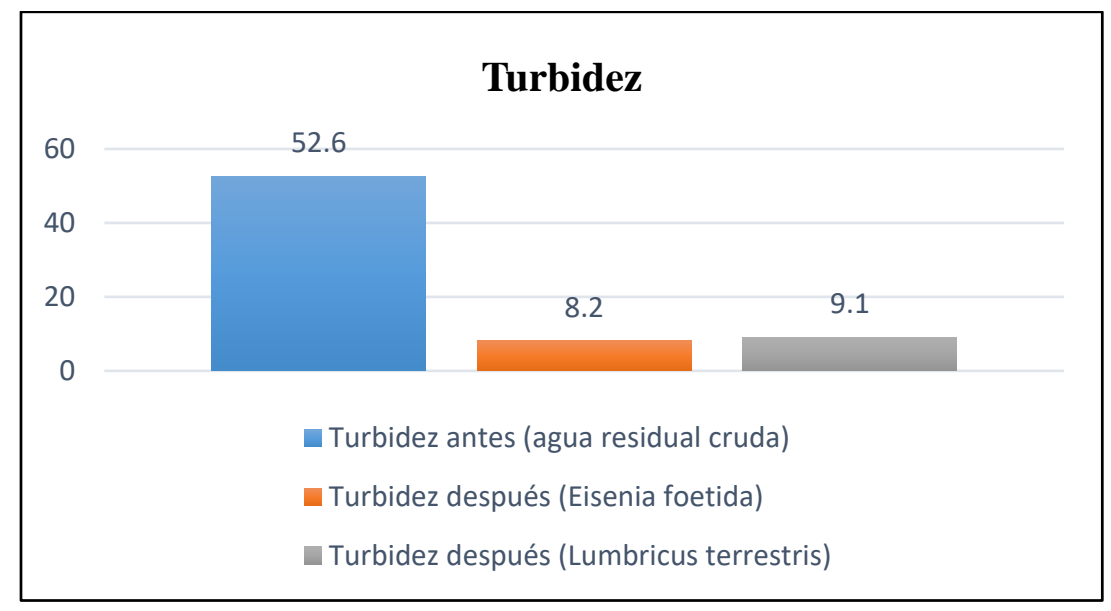

Figura 5. Comparación del parámetro Turbidez antes y después del tratamiento mediante el lombrifiltro con las especies Eisenia foetida y Lumbricus terrestris

\section{Potencial de hidrógeno (pH)}

En un inicio el agua residual doméstica se encontraba con un $\mathrm{pH}$ ácido de 6.2, pero después del tratamiento mediante el lombrifiltro el resultado cambio y fue un $\mathrm{pH}$ estable de 6.7 con la especie Eisenia foetida y 6.68 con la especie Lumbricus terrestris (figura 6), el cual es óptimo para el crecimiento de bacterias nitrificantes ya que estas crecen en ambientes con $\mathrm{pH}$ entre 6.5 a 8.6, y este reporte acredita y concuerda con el resultado de la remoción del nitrógeno de un $77 \%$ y $78 \%$ en eficiencia, presentada anteriormente en la presente investigación.

Asimismo, se pudo observar que el pH iba en aumento; esto se debía a que las lombrices (para ambas especies) tienen la capacidad de estabilizar la acidez del agua gracias a sus glándulas calcíferas que se encuentran en el esófago de esta. Pero ¿cómo es el proceso? cuando el material orgánico llega al estómago, unas glándulas especiales se encargan de segregar carbonato cálcico, cuya finalidad es neutralizar los ácidos presentes en la comida ingerida, la que después de atravesar todo el aparato digestivo es expulsado 
por el ano (defecando), que se encuentra en la parte terminal, de esta manera va añadiendo poco a poco calcio, haciendo indirectamente que el agua residual se equilibre en el $\mathrm{pH}$.

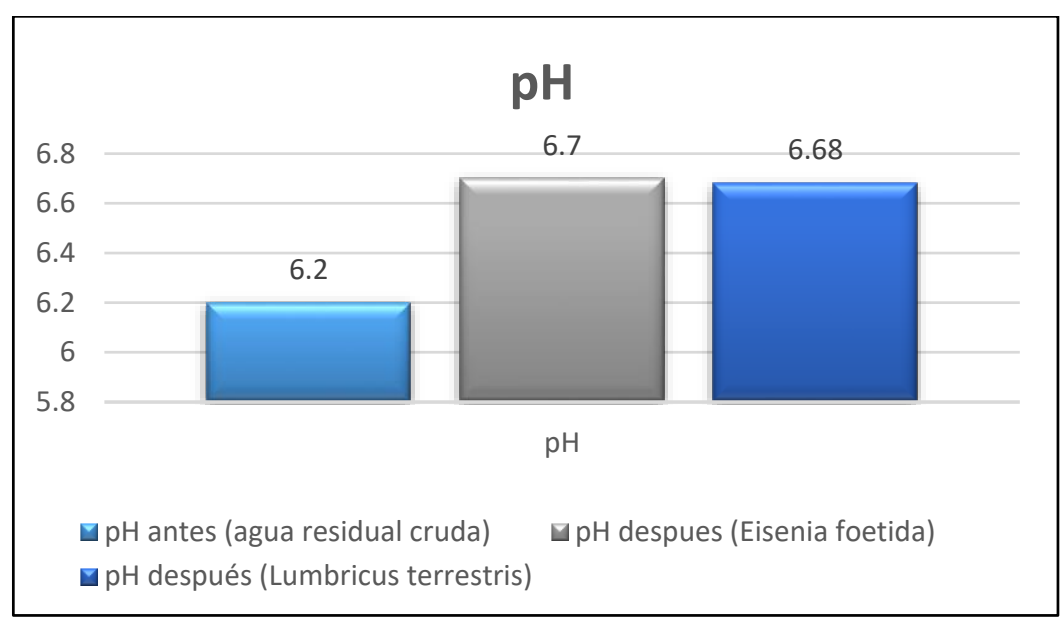

Figura 6. Comparación del parámetro $\mathrm{pH}$ antes y después del tratamiento mediante el lombrifiltro con las especies Eisenia foetida y Lumbricus terrestris

\section{Coliformes termotolerantes}

La cantidad de Coliformes termotolerantes antes del ingreso al tratamiento mediante el lombrifiltro fue $4200 \mathrm{NMP} / 100 \mathrm{~mL}$, después de pasar por el sistema de depuración se obtuvo un valor de $685 \mathrm{NMP} / 100 \mathrm{~mL}$ con la especie Eisenia foetida mostrando una eficiencia de $84 \%$, sin embargo, con la especie Lumbricus terrestris se tuvo un resultado de $820 \mathrm{NMP} / 100 \mathrm{~mL}$ con una eficiencia de $80 \%$ (figura 7). La notable y óptima remoción se puede atribuirse a las diversas acciones y sustancias generadas por las lombrices y microorganismos consumidores de materia orgánica, que viven junto con las lombrices, tales como la acción enzimática intestinal, secreción de los celomas estos son fluidos que tienen propiedades antibacterianas, y el pastoreo selectivo que estos realizan (Monte, et al., 2014). 


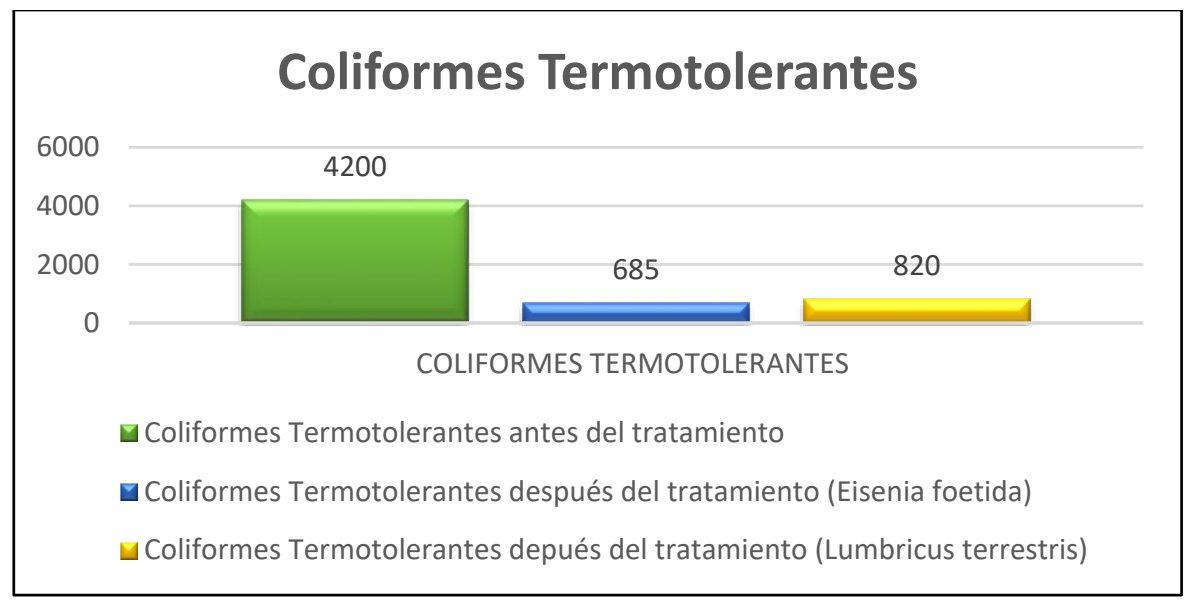

Figura 7. Comparación del parámetro $\mathrm{pH}$ antes y después del tratamiento mediante el lombrifiltro con las especies Eisenia foetida y Lumbricus terrestris

\section{Determinación de la eficiencia del lombrifiltro según especie en estudio}

Para evaluar el funcionamiento del prototipo biológico compuesto por dos tipos de especie (Eisenia foetida y Lumbricus terrestris) se determinó el porcentaje de eficiencia de los parámetros analizados (tabla 11).

\section{Tabla 11}

Eficiencia del método del lombrifiltro

\begin{tabular}{lcc}
\hline \multicolumn{1}{c}{ Parámetros } & $\begin{array}{c}\text { Eficiencia- } \boldsymbol{E F} \\
(\boldsymbol{\%})\end{array}$ & $\begin{array}{c}\text { Eficiencia- } \boldsymbol{L T} \\
(\boldsymbol{\%})\end{array}$ \\
\hline DBO5 & 92 & 91 \\
DQO & 86 & 84 \\
Nitrógeno & 78 & 77 \\
turbidez & 84.4 & 83 \\
pH & 93 & 93 \\
Coliformes & 84 & 80 \\
termotolerantes & & \\
Promedio total de & 87 & 85 \\
eficiencia & 87 & \\
\hline
\end{tabular}

\section{Resultados estadísticos de contraste de hipótesis}

Análisis estadístico a través de Prueba de wilcoxon para

los Parámetros físico-químicos y microbiológicos

de la especie Eisenia foetida

Para el análisis de la prueba wilcoxon respecto a la especie Eisenia foetida se realizaron las siguientes formulaciones de hipótesis: 
- Ho1: El método del lombrifiltro con la especie Eisenia foetida es eficiente en la remoción de contaminantes fisicoquímicos y microbiológicos del agua residual doméstica.

- Ha: El método del lombrifiltro con la especie Eisenia foetida no es eficiente en la remoción de contaminantes fisicoquímicos y microbiológicos del agua residual doméstica.

Ante ello se obtuvo los siguientes resultados descritos a continuación en la Tabla 12:

Tabla 12

Prueba estadística de Wilcoxon para los contaminantes fisicoquímicos y microbiológicos para la especie Eisenia foetida

\begin{tabular}{ccccccc}
\hline Wilcoxon & pH & Turbidez & DBO5 & DQO & Nitrógeno & CT \\
\hline Z & -3.464 & -7.746 & -11.00 & -16.91 & -9.055 & -69.886 \\
$\begin{array}{c}\text { Sig. asintótica } \\
\text { (bilateral) }\end{array}$ & $\mathbf{0 . 0 0 1}$ & $\mathbf{0 . 0 0 0}$ & $\mathbf{0 . 0 0 0}$ & $\mathbf{0 . 0 0 0}$ & $\mathbf{0 . 0 0 0}$ & $\mathbf{0 . 0 0 0}$ \\
\hline
\end{tabular}

Fuente: Extraída del SPSS 24.0

\section{$\underline{\text { Regla de decisión }}$}

Como el valor del $\operatorname{sig}=0,000<\alpha=0,05$ para todos los contaminantes fisicoquímicos y microbiológicos, se decide rechazar la hipótesis nula a favor de la alternativa.

\section{Conclusión}

El método del lombrifiltro con la especie Eisenia foetida fue eficiente en la remoción de los contaminantes fisicoquímicos y microbiológicos de las aguas residuales domésticas, demostrando de esta manera que el método contribuye favorablemente y otorga un aporte significativo al Distrito de Chachapoyas-Amazonas.

\section{Análisis estadístico a través de Prueba de Wilcoxon para los parámetros físico- químicos y microbiológicos de la especie Lumbricus terrestris}

Para el análisis de la prueba wilcoxon respecto a la especie Lumbricus terrestris se realizaron las siguientes formulaciones de hipótesis: 
- Ho2: El método del lombrifiltro con la especie Lumbricus terrestris es eficiente en la remoción de contaminantes físicoquímicos y microbiológicos del agua residual doméstica

- Ha2: El método del lombrifiltro con la especie Lumbricus terrestris no es eficiente en la remoción de contaminantes fisicoquímicos y microbiológicos del agua residual doméstica

Ante ello se obtuvo los siguientes resultados descritos a continuación en la Tabla 13:

\section{Tabla 13}

Prueba estadística de Wilcoxon para los contaminantes fisicoquímicos y microbiológicos de la especie Lumbricus terrestris

\begin{tabular}{ccccccc}
\hline Wilcoxon & pH & Turbidez & DBO5 & DQO & Nitrógeno & CT \\
\hline Z & -3.464 & -7.810 & -11.045 & -17.059 & -9.110 & -70.845 \\
$\begin{array}{c}\text { Sig. asintótica } \\
\text { (bilateral) }\end{array}$ & $\mathbf{0 . 0 0 1}$ & $\mathbf{0 . 0 0 0}$ & $\mathbf{0 . 0 0 0}$ & $\mathbf{0 . 0 0 0}$ & $\mathbf{0 . 0 0 0}$ & $\mathbf{0 . 0 0 0}$ \\
\hline
\end{tabular}

Fuente: Extraída del SPSS 24.0

\section{Regla de decisión}

Como el valor del $\operatorname{sig}=0,000<\alpha=0,05$ para todos los contaminantes fisicoquímicos y microbiológicos para la especie Lumbricus terrestris, se decide rechazar la hipótesis nula a favor de la alternativa.

\section{Conclusión}

El método del lombrifiltro con la especie Lumbricus terrestris fue eficiente en la remoción de los contaminantes físicoquímicos y microbiológicos de las aguas residuales domésticas, demostrando de esta manera que el método contribuye favorablemente y otorga un aporte significativo al el Distrito de ChachapoyasAmazonas.

\section{Análisis estadístico a través de Prueba de U Mann - Whitney para los parámetros físico-químicos y microbiológicos de las especies Eisenia foetida $y$ Lumbricus terrestres}

Para el análisis de la U Mann-Whitney respecto a las especies Eisenia foetida y Lumbricus terrestris se realizaron las siguientes formulaciones de hipótesis: 
- Ho3: El método del lombrifiltro con la especie Eisenia foetida difiere de la especie Lumbricus terrestris en la remoción de contaminantes fisicoquímicos y microbiológicos del agua residual doméstica.

- Ha3: El método del lombrifiltro con la especie Eisenia foetida no difiere de la especie Lumbricus terrestris en la remoción de contaminantes fisicoquímicos y microbiológicos del agua residual doméstica.

\section{Tabla 14}

Análisis comparativo de los parámetros fisicoquímico y microbiológicos según especie a través de la prueba de U de Mann-Whitney

\begin{tabular}{ccccccc}
\hline Personalización & $\begin{array}{c}\text { Valores } \\
\text { pH }\end{array}$ & $\begin{array}{c}\text { Valores } \\
\text { Turbidez }\end{array}$ & $\begin{array}{c}\text { Valores } \\
\text { DBO5 }\end{array}$ & $\begin{array}{c}\text { Valores } \\
\text { DQO }\end{array}$ & $\begin{array}{c}\text { Valores } \\
\text { Nitrógeno }\end{array}$ & $\begin{array}{c}\text { Valores } \\
\text { CT }\end{array}$ \\
\hline $\begin{array}{c}\text { U de Mann- } \\
\text { Whitney }\end{array}$ & 0,000 & 0,000 & 0,000 & 0,000 & 0,000 & 0,000 \\
$\begin{array}{c}\text { Z } \\
\text { Sig. Asintótica } \\
\text { (bilateral) }\end{array}$ & -3.606 & -4.000 & -4.472 & -8.718 & $-5,477$ & $-38,781$ \\
\hline Funden & 0.000 & 0,000 & 0,000 & 0,000 & 0,000 & 0,000 \\
\hline
\end{tabular}

Fuente: Extraída del SPSS 24.0

\section{$\underline{\text { Regla de decisión }}$}

Como el valor del sig $=0,000<\alpha=0,05$ para todos los parámetros fisicoquímicos y microbiológicos, se decide rechazar la hipótesis nula para todos los casos a favor de la alternativa.

\section{Conclusión}

El lombrifiltro demostró una efectiva remoción en todos los parámetros analizados, alcanzando eficiencias del $92 \%$ con la especie Eisenia foetida y $91 \%$ con la Lumbricus terrestris respecto a la demanda biológica de oxígeno (DBO5), la demanda química de oxígeno (DQO) fue de 86\% EF y 84\% LT; en el Nitrógeno total; 78\% EF y 77\% LT; en la turbidez; $84.4 \%$ EF y $83 \%$ LT; de Coliformes Termotolerantes $84 \%$ EF y $80 \%$ LT; asimismo, se obtuvo un $\mathrm{pH}$ neutro de 6.7 y 6.68 con un porcentaje de eficiencia del 93\% para ambas especies respectivamente.

El diseño del método de tratamiento se realizó con los datos que influyen directamente en el resultado como; el caudal que fue $148.3 \mathrm{~mL} / \mathrm{min}$ un caudal que permitió la alimentación exacta para lombrices; asimismo el parámetro DBO5 inicial (112.3 mg/L) escogido dentro de tres horarios diferentes, además de las dimensiones que fueron de un largo de $0.45 \mathrm{~m}$ un ancho de $0.25 \mathrm{~m}$ y una altura de $0.30 \mathrm{~m}$. 
Los actores principales en la degradación de la materia orgánica fueron las especies EF y LT la elección de estas especies fue debido a su alta eficiencia en la remoción de los contaminantes sobre todo en la materia orgánica, se adapta rápidamente en el entorno o medio y pueden cohabitar aglomeradas entre sí para la EF, a diferencia de la LT fue un reto importante en la investigación porque de esta forma se determinó su eficiencia respecto a la remoción de los contaminantes del agua residual doméstica, esta especie fue elegida por su fácil accesibilidad y no tiene costo alguno porque se lo puede encontrar en todo tipo de tierras húmedas.

Tanto los parámetros físicoquímicos como microbiológico se encuentran por debajo de los valores establecidos por los Decreto Supremo N ${ }^{\circ}$ 004-2017-MINAM, encontrándose ambas especies dentro de los estándares permitido por el decreto. Concluyendo así, que dicha agua es apta para ser utilizada en el regadío de vegetales no restringido y restringido, como para bebidas de animales, haciéndola una opción efectiva para el tratamiento de las aguas residuales domesticas de la ciudad de Chachapoyas. Asimismo, el efluente no tenía ningún olor desagradable y tampoco había proliferación de vectores.

Finalmente, la eficiencia de remoción de contaminantes de las aguas residuales domesticas de la ciudad de Chachapoyas, fue comparada entre especies teniendo que la EF obtuvo una remoción promedio del 87\%, mientras que la LT lo hizo en un $85 \%$, demostrándose en esta investigación que la especie EF es más eficiente para la remoción de contaminantes, sin embargo, ambas especies reflejan una buena eficiencia debido a que encuentran dentro de los límites aceptables por el DS vigente.

\section{Referencias}

Arenas, S., \& Nuncira, A. (2010). Evaluación de Humedales Artificiales para el Tratamiento de Aguas Residuales del Sector Industrial Avicola. Universidad Industrial de Santander Facultad.

Brack, A., Aranda, C., Bernales, M., Bustamante, R., Campos, L., Capurro, F., Gomero, L. (2008). Diagnóstico ambiental del Perú. Lima.

Jiang, L., Liu, Y., Hu, X., Zeng, G., Wang, H., \& Zhou, L. (2016). The use of microbialearthworm ecofilters for wastewater treatment with special attention to influencing factors in performance: A review. Bioresource Technology, 200, 999-1007. https://doi.org/10.1016/j.biortech.2015.11.011

Lorenzo, E., Llanes, J., Fernández, L., \& Bataller, M. (2009). Reúso de aguas residuales domésticas para riego agrícola. Revista CENIC Ciencias Biológicas, 40(1), 35-44.

Madueño, R., \& Sandoval, J. (2009). Evaluación del uso de la planta acuática Lemna minor para determinar la eficiencia remocional de nutrientes a escala reactor del 
efluente de la laguna Secundaria de la Planta CITRAR. Universidad Nacional de Ingeniería.

Martinez, C. (2012). Lombricultura. Mexico.

MINAM. (2012). Política Nacional de Educación Ambiental. Lima.

Monte, M., Ruiz, G., Saavedra, L., \& Suarez, F. (2014). Tratamiento de Aguas Servidas Alternativo : Lombrifiltro.

Nodal, E. (2001). Procesos Biológicos Aplicados al Tratamiento de Agua Residual, XXII(4), 52-56.

OEFA. (2016). INFOR. 0459-2016-OEFA-DS-SEP MP CHACHAPOYAS.pdf. Chachapoyas.

Pérez, A. (2010). Selección de sistema de tratamiento de aguas residuales para localidad de Santa Barbara usando metodología de decisión multicriterio AHP. Universidad de Chile.

Ramón, J., Leon, J., \& Castillo, N. (2015). Diseño de un sistema alternativo para el tratamien - to de aguas residuales urbanas por medio de la técnica de lombrifiltros utilizando la especie Eisenia foetida. Mutis, 5(1), 46-54.

Sampieri, R., Fernández, C., \& Baptista, L. (2010). Metodología de la investigación. México: McGraw-Hill.

SUNASS. (2015). Benchmarking Regulatorio de la EPS.

SUNASS. (2014). Benchmarking Regulatorio de las EPS (Datos 2014) 2014.

Wang, L., Luo, X., Zhang, J., \& Zhen, Z. (2011). Performance and mechanisms of a microbial-earthworm ecofilter for removing organic matter and nitrogen from synthetic domestic wastewater. Journal of Hazardous Materials, 195(June 2016), 245-253. https://doi.org/10.1016/j.jhazmat.2011.08.035 